\section{Kühlung der Proben}

W. G. Guder

München, Deutschland

Englischer Begriff cooling of samples; refrigeration of samples

Definition Eine Kühlung der Probe liegt dann vor, wenn sie nach Gewinnung auf Temperaturen unterhalb der Raumtemperatur gehalten wird. Man unterscheidet dabei Kühlschranktemperaturen $\left(2-8{ }^{\circ} \mathrm{C}\right)$ von gefrorenem (unter -5 bis $-6{ }^{\circ} \mathrm{C}$ ) und tiefgefrorenem Zustand (unter $-20^{\circ} \mathrm{C}$ ).

Beschreibung Eine Kühlung der Probe (Untersuchungsmaterial Blut, Urin, Liquor etc.) ist dann nach Gewinnung empfohlen, wenn die Aufbewahrung der Probe zur erhöhten Stabilität des zu messenden Analyten beiträgt. Dabei sind je nach gewünschter Untersuchung verschiedene Regeln einzuhalten:

Vollblut sollte nicht gekühlt werden, weil dieser Vorgang die metabolischen Prozesse der Zellen hemmt und dadurch den Austausch der Elektrolyte zwischen Zellen und Plasma eher beschleunigt $(\triangleright$ Kalium, $\triangleright$ Chlorid, ionisiertes $\triangleright$ Cal- cium). Andererseits ist eine Stabilisierung des Stoffwechsels Grundvoraussetzung zur Erhaltung der Blutgase, des $\mathrm{pH}$ und der $\triangleright$ Glukose, wenn die Zeit über 15 Minuten hinausgeht. Angaben zur Stabilität aller Analyten finden sich in der angegebenen Literatur.

Einige Analyten vertragen aus verschiedenen Gründen keine Kühlung unter Kühlschranktemperatur: Lipoproteine, hämatologische Proben, Urin-IgG, \ Harnsediment und • Harnsäure im Urin.

Beim Auftauen ist zu berücksichtigen, dass sich während der Kühlung eine Inhomogenität der Analyten einstellt. Diese muss beim Auftauen durch vorsichtiges Mischen vor Abnahme der analytischen Probe wieder aufgelöst werden. Bei auskristallisierten Bestandteilen ist eine vorsichtige Erwärmung zur Wiederlösung des Analyten zu empfehlen.

\section{Literatur}

Guder WG, Narayanan S, Wisser H, Zawta B (2009) Diagnostic samples: from the patient to the laboratory, 4. Aufl. Wiley-Blackwell, Weinheim

Guder WG, da Fonseca-Wollheim F, Heil W et al (2012) Die Qualität diagnostischer Proben, 7. Aufl. BD Diagnostics, Heidelberg 